\title{
Reducing BER of spectral-amplitude coding optical code-division multiple-access systems by single photodiode detection technique
}

H. M. R. Al-Khafaji

eng.hamza.alkhafajigmail.com

\section{S. A. Aljunid}

\section{A. Amphawan}

\section{H. A. Fadhil}

\section{A. M. Safar}

\begin{abstract}
School of Computer and Communication Engineering, University Malaysia Perlis (UniMAP), Perlis, Malaysia

School of Computer and Communication Engineering, University Malaysia Perlis (UniMAP), Perlis, Malaysia

Internetworks Research Group, School of Computing, University Utara Malaysia (UUM), Kedah, Malaysia

School of Computer and Communication Engineering, University Malaysia Perlis (UniMAP), Perlis, Malaysia

School of Computer and Communication Engineering, University Malaysia Perlis (UniMAP), Perlis, Malaysia
\end{abstract}

In this paper, we present a single photodiode detection (SPD) technique for spectral-amplitude coding optical code-division multiple-access (SAC-OCDMA) systems. The proposed technique eliminates both phase-induced intensity noise (PIIN) and multiple-access interference (MAI) in the optical domain. Analytical results show that for 35 simultaneous users transmitting at data rate of 622 Mbps, the bit-error rate $(B E R)=1.4 \times 10^{-28}$ for SPD technique is much better compared to $9.3 \times 10^{-6}$ and $9.6 \times 10^{-3}$ for the modified-AND ${ }^{a}$ as well as the $\mathrm{AND}^{a}$ detection techniques, respectively. Moreover, we verified the improved performance afforded by the proposed technique using data transmission simulations. [DOI: http://dx.doi.org/10.2971/jeos.2013.13022]

${ }^{a}$ The modified-AND as well as the AND are the names of subtraction detection techniques that are proposed in the literature.

Keywords: Optical code-division multiple-access, spectral-amplitude coding, single photodiode detection technique, multiple-access interference, phase-induced intensity noise

\section{INTRODUCTION}

Over the past several years, optical code-division multipleaccess (OCDMA) systems have been proven to be very helpful in combining the unlimited bandwidth of fiber with the flexibility of CDMA technique to achieve high-quality transmission without traffic management or system synchronization. However, the main degradation on the performance of OCDMA systems is essentially due to MAI originating from other simultaneous users, as this severely increases the likelihood of bit errors occurring [1]. Hence, spectral-amplitude coding (SAC) garners significant attention because of its ability to combat MAI by employing an appropriate subtraction detection, and the availability of cost-effective broadband sources, such as light-emitting diodes (LEDs) [2]. Even so, systems employing these broadband sources are PIIN-limited owing to the thermal-like nature of incoherent light $[3,4]$. Therefore, the summation of different users' signals at the receiver's side fluctuate in intensity, which increases the distortion of the signal received [5]. Due to this, PIIN reduction is a matter of considerable importance in incoherent SAC-OCDMA systems.

The most common techniques used to extract user signals in the presence of other interferers are AND subtraction detection [6] and complementary subtraction detection [7]. The AND subtraction detection technique reduces the number of filters used by the complementary subtraction detection technique and thus further reduces the receiver's insertion losses and cost. The modifiedAND subtraction detection technique was recently proposed to minimize PIIN and MAI in incoherent SACOCDMA systems by dividing the spectrum of the decoded signals [8]. The modified-AND subtraction detection technique has the same receiver complexity as the AND detection technique while enhancing system performance. All of these techniques utilise balanced detection, composed of two photodiodes connected electrically in opposition. The output signal is proportional to the power difference between the two optical inputs. As such, it would be valuable to design a detection scheme using a single photodiode instead of two. The reduction in the number of photodiodes will reduce the cost of the receiver, as well as the generated shot noise. The paper is organised into four sections. After Introduction in Section, Section 2 provides a detailed explanation of the SPD technique. Next, in Section 3, we focus on performance analysis followed by the discussion of the results. Finally, Section 4 summarises our conclusions. 


\section{SINGLE PHOTODIODE DETECTION (SPD) TECHNIQUE}

The SAC-OCDMA receiver diagram of this technique is shown in Figure 1. The incoming optical signal is decoded by the decoder, which has an identical spectral response to the intended encoder for the data to be received. The detected output from the decoder is either $w$ power units (P.U.) for active user or $\lambda$ P.U. for interferers, where the weight $w$ representing the number of occupied frequency bins in the user's encoder, and the in-phase cross-correlation $\lambda$, which is the maximum number of common frequency bins occupied by any two codes of the family [9]. The remainder of the signal from the decoder is then transmitted to the subtractive decoder (s-Decoder) to cancel out signals with mismatched signatures, i.e., interferers. The s-Decoder contains only frequency bins from different interferers represented logically in Table 1. The output from the $s$-Decoder is either zero P.U. for active user or $\lambda$ P.U. for interferers. This technique can be performed using inexpensive fiber Bragg-gratings (FBGs) to decode the received signal. After optical subtraction, the output is either $w$ P.U. for active user or zero P.U. for interferers. This implies that the interference signals are cancelled in the optical domain before the conversion of the signals to the electrical domain, as a consequence, the new SPD scheme alleviates both PIIN and MAI in the optical domain. Theoretically, the two interference signals at the optical subtractor are assumed to be equal and cancel each other out. However, in practice, the interference signals differ slightly at the optical subtractor and results in a small amount of optical power to reach the photodiode (PD). This limitation not only prevails in the new SPD technique, but also in other electrical subtraction detection techniques. The cancellation of the interference signals in the optical domain allows the use of only a single photodiode rather than two photodiodes as in typical subtraction detection schemes. This reduces the amount of optical-to-electrical conversion and shot noise generated at the receiver. This method can also be implemented with any fixed in-phase cross-correlation code with a simple modification in the spectral distribution of the s-Decoder, depending on the construction of the code used. After the desired signal is detected by a photodiode, the data-carrying electrical signal is low pass-filtered by a Bessel-Thompson filter of order four to reject the out-of-band high-frequency noise. The bandpass resulting from the filtering $\left(B_{e}\right)$ is assumed to be equivalent to $0.75 \times R_{B}$, where $R_{B}$ is the data rate $[5,9]$.

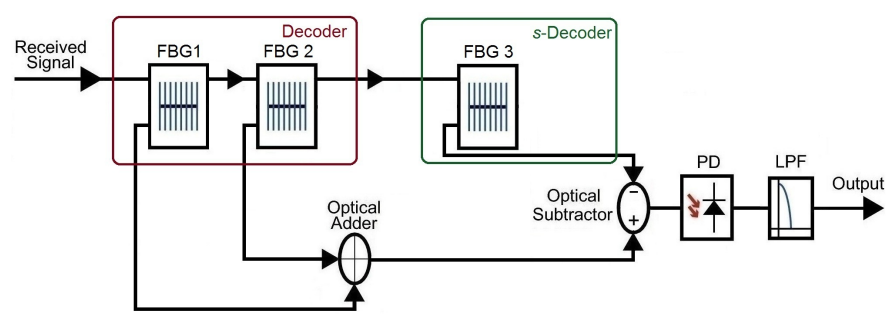

FIG. 1 SAC-OCDMA receiver based on SPD technique. FBG: fiber Bragg-grating; PD: photodiode; LPF: low pass filter.

\begin{tabular}{|c|c|}
\hline & Code words \\
\hline Main User (DEC) & $\{1101100000\}$ \\
\hline $1^{\text {st }}$ Interfering User $\left(I_{1}\right)$ & $\{011000110\}$ \\
\hline $2^{\text {nd }}$ Interfering User $\left(I_{2}\right)$ & $\{00000110011\}$ \\
\hline$\left(\mathrm{DEC} \bullet I_{1}\right)$ & 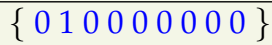 \\
\hline$\overline{\Sigma\left(I_{1} \bullet \mathrm{DEC}\right)}$ & 1 \\
\hline$\overline{D E C}$ & $\{001001111\}$ \\
\hline$\left(I_{1} \bullet I_{2}\right)$ & 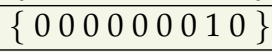 \\
\hline$s$-DEC $=\overline{\mathrm{DEC}} \bullet\left(I_{1} \bullet I_{2}\right)$ & 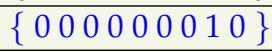 \\
\hline$\left(I_{1} \bullet s-\mathrm{DEC}\right)$ & $\left\{\begin{array}{llllllll}0 & 0 & 0 & 0 & 0 & 0 & 10 & 0\end{array}\right.$ \\
\hline$\Sigma\left(I_{1} \bullet s-\mathrm{DEC}\right)$ & 1 \\
\hline$\Sigma\left(I_{1} \bullet \mathrm{DEC}\right)-\Sigma\left(I_{1} \bullet s-\mathrm{DEC}\right)$ & $1-1=0$ \\
\hline
\end{tabular}

TABLE 1 Logical representation of interference cancellation for MDW code.

\section{PERFORMANCE ANALYSIS AND RESULTS}

In this section, the analytical expressions for the mean optical power and major noises are presented in order to compute the BER performance of SAC-OCDMA system based on SPD technique. Modified double-weight (MDW) codes are utilised as the signature sequences for SAC-OCDMA systems. MDW codes are characterised by unity cross-correlation $(\lambda=$ $1)$, which is the ideal cross-correlation value. For a weight of four $(w=4)$, the code length is: [10]

$$
L=3 K+\frac{8}{3}\left[\sin \left(\frac{K \pi}{3}\right)\right]^{2}
$$

where $L$ is the length of the code, and $K$ is the number of users. The mean optical power reaching PD when the desired user is active is given by: [9]

$$
\begin{aligned}
& \left\langle P_{\text {user }}\right\rangle=S\left[\int_{0}^{B_{o}} H_{E}(v) H_{D}(v) d v\right] \\
& \left\langle P_{\text {user }}\right\rangle=S \frac{B_{o}}{L} \sum_{i=1, E=D}^{L} c_{E}(i) \bullet c_{D}(i) \\
& \left\langle P_{\text {user }}\right\rangle=\frac{S B_{o}}{L} w=\frac{4 S B_{o}}{L}
\end{aligned}
$$

where $S$ is the received power spectral density level at each $\mathrm{PD}$ and it is assumed to be equal for mathematical simplicity [11], $B_{0}$ is the optical bandwidth partitioned into logical frequency bins of width $B_{o} / L, H_{E}(v)$ and $H_{D}(v)$ are the encoder and decoder transfer functions, respectively. These functions are spectral representations of the filters. They are made of bins of a given spectral width that are fully transmissive or fully opaque, and $c_{E}(i)$ and $c_{D}(i)$ denote the $i^{\text {th }}$ element of the encoder and decoder code words, respectively.

The PIIN expression for unpolarized thermal light source can be expressed as follows: [9]

$$
\begin{aligned}
\sigma_{\text {PIIN }}^{2} & =S^{2} B_{e} \int_{0}^{B_{o}}\left[H_{E}(v) H_{D}(v)\right]^{2} d v \\
\sigma_{\text {PIIN }}^{2} & =S^{2} B_{e} \frac{B_{o}}{L} \sum_{i=1, E=D}^{L}\left[c_{E}(i)\right]^{2} \bullet\left[c_{D}(i)\right]^{2} \\
\sigma_{\text {PIIN }}^{2} & =\frac{S^{2} B_{e} B_{o}}{L} w=\frac{4 S^{2} B_{e} B_{o}}{L}
\end{aligned}
$$


while the variance of shot noise can be evaluated as: [12]

$$
\sigma_{\text {sh }}^{2}=2 e B_{e} \Re\left\langle P_{u s e r}\right\rangle
$$

The variance of thermal noise is also given by: [12]

$$
\sigma_{t h}^{2}=\frac{4 k_{B} T_{n} B_{e}}{R_{L}}
$$

and the signal-to-noise ratio is thus

$$
S N R=\frac{\left(\left\langle P_{U s e r}\right\rangle\right)^{2}}{\sigma_{P I I N}^{2}+\sigma_{s h}^{2}+\sigma_{t h}^{2}}
$$

Based on the approximation of Gaussian distribution, the BER is given by: [8]

$$
B E R=\frac{1}{2} \operatorname{erfc}\left(\frac{\sqrt{S N R}}{2}\right)
$$

where $\operatorname{erfc}$ is the complementary error function. Table 2 illustrates the typical parameters used for our calculations.

Figure 2 shows the variation of the BER versus the number of active users (K) for the SPD (at different data rates) against AND as well as modified-AND detection schemes. The BER of the SAC-OCDMA system increases when the number of active users increases, mainly due to PIIN. Nonetheless, the SPD scheme is capable of achieving superior BER performance over other detection approaches and gives the advantage of increasing the number of active users without affecting the quality-of-service (QoS). It is shown that for $K=35$ and data rate of $622 \mathrm{Mbps}$, the $\mathrm{BER}=1.4 \times 10^{-28}$ for SPD technique is much better compared to $9.3 \times 10^{-6}$ and $9.6 \times 10^{-3}$ for the modified-AND as well as the AND detection techniques, respectively. Furthermore, interestingly, the BER performance of the SPD scheme at data rate of $1.25 \mathrm{Gbps}$ is better than other detection schemes at data rate of $622 \mathrm{Mbps}$.

We confirm the validity of the proposed scheme by simulating three channels of MDW code $(w=4)$ using OptiSystem software (Version 9.0) from optiwave ${ }^{T M}$. Simulations were implemented using one LED sliced into 12 wavelengths, which are code 1: $(1550 \mathrm{~nm}),(1550.8 \mathrm{~nm}),(1552.4 \mathrm{~nm}),(1553.2 \mathrm{~nm})$, code 2: $(1550.8 \mathrm{~nm}),(1551.6 \mathrm{~nm}),(1554.8 \mathrm{~nm}),(1555.6 \mathrm{~nm})$, and code 3: (1553.2 $\mathrm{nm}),(1554 \mathrm{~nm}),(1555.6 \mathrm{~nm}),(1556.4 \mathrm{~nm})$. Each chip has a spectral width of $0.8 \mathrm{~nm}$. The information signals are generated using a $2^{14}-1$ pseudo random bit sequence (PRBS) generator with the non-return-to-zero (NRZ) line coding before being modulated with the codes using an external Mach-Zehnder modulator (MZM). The attenuation and the dispersion coefficients of single mode fiber (SMF) at a wavelength of $1550 \mathrm{~nm}$ are $0.25 \mathrm{~dB} / \mathrm{km}$ and $18 \mathrm{ps} / \mathrm{nm} \mathrm{km}^{-1}$ respectively. FBGs with uniform apodisation function are used

\begin{tabular}{|l|l|}
\hline Optical bandwidth & $\mathrm{B}_{o}=3.75 \mathrm{THz}$ \\
\hline Electron's charge & $e=1.602 \times 10^{-19} \mathrm{C}$ \\
\hline Responsivity of the photodiode & $\Re=0.75$ \\
\hline Boltzmann's constant & $\mathrm{k}_{B}=1.38066 \times 10^{-23} \mathrm{JK}^{-1}$ \\
\hline Receiver noise temperature & $\mathrm{T}_{n}=300 \mathrm{~K}$ \\
\hline Receiver load resistance & $\mathrm{R}_{L}=1030 \Omega$ \\
\hline
\end{tabular}

TABLE 2 Typical parameters used in simulations.

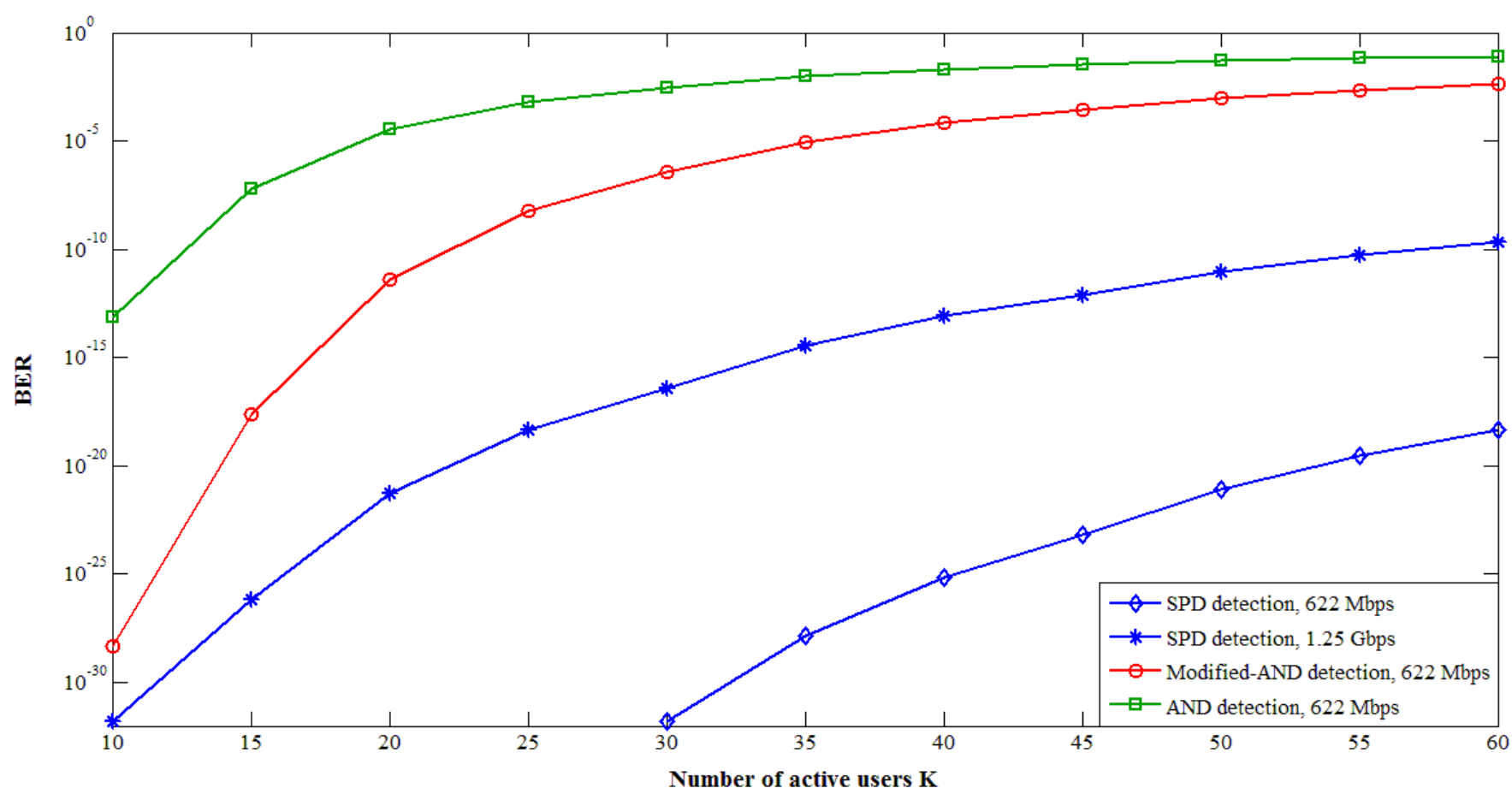




\begin{tabular}{|c|c|c|c|}
\hline User & FBG & $\begin{array}{c}\text { Center wavelength } \\
\text { (nm) }\end{array}$ & $\begin{array}{c}\text { Optical bandwidth } \\
\text { (nm) }\end{array}$ \\
\hline \multirow{3}{*}{ User 1 } & FBG 1 & 1550.4 & 1.6 \\
& FBG 2 & 1552.8 & 1.6 \\
& FBG 3 & 1555.6 & 0.8 \\
\hline \multirow{3}{*}{ User 2 } & FBG 1 & 1551.2 & 1.6 \\
& FBG 2 & 1555.2 & 1.6 \\
& FBG 3 & 1553.2 & 0.8 \\
\hline \multirow{3}{*}{ User 3 } & FBG 1 & 1553.6 & 1.6 \\
& FBG 2 & 1556 & 1.6 \\
& FBG 3 & 1550.8 & 0.8 \\
\hline
\end{tabular}

TABLE 3 FBGs characteristics used in simulations.

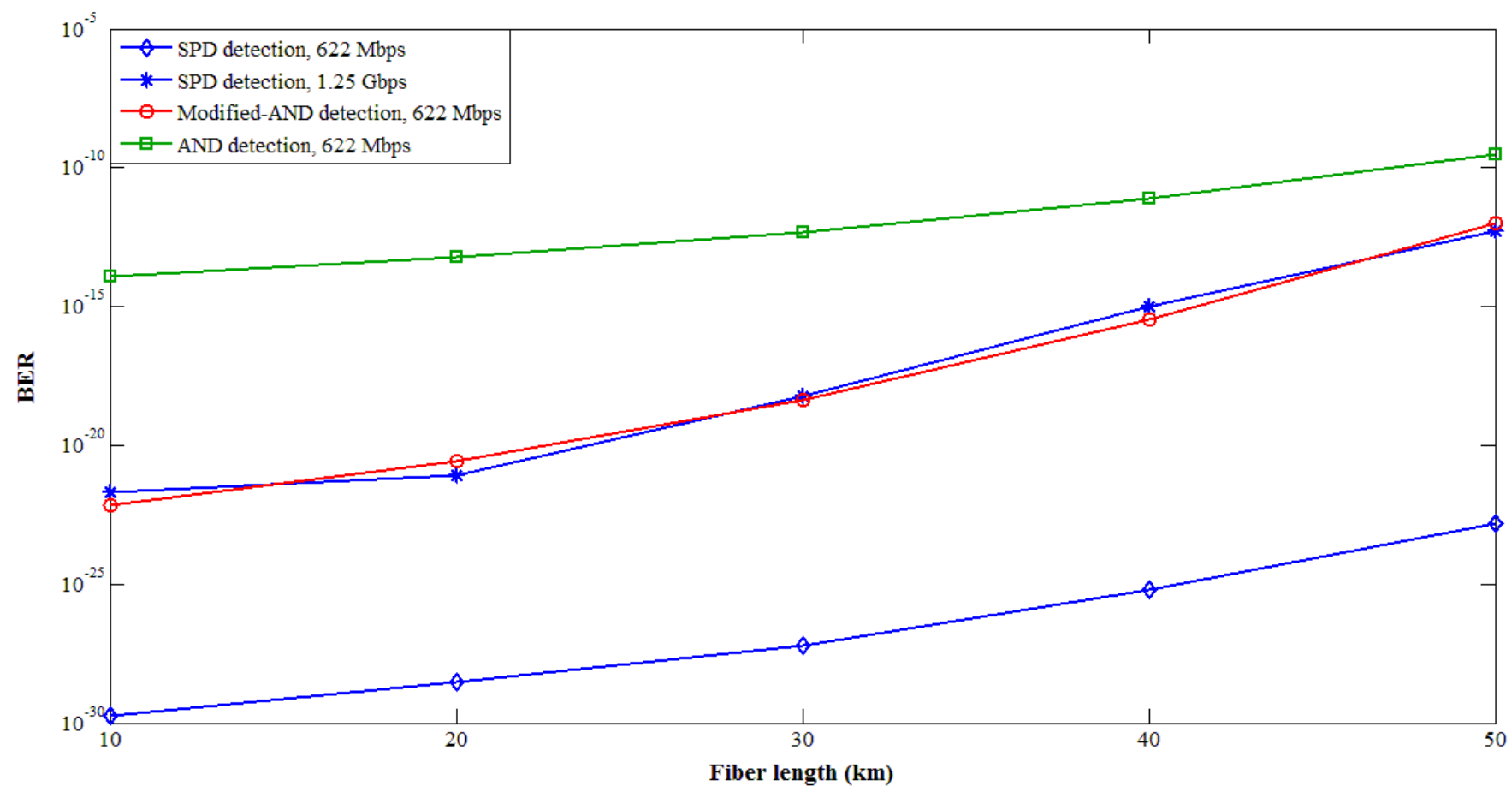

FIG. 3 BER versus fiber length for different detection techniques.

to decode the received signal. The center wavelength and optical bandwidth for FBGs of each user are listed in Table 3, where FBG1 and FBG2 representing the decoder, and FBG3 represents the s-Decoder. FBG1 and FBG2 with bandwidth twice the chip width and average adjacent center wavelengths are employed to decode the desired user's signal. The optical subtractor component is an ideal model. The dark current value was set at $5 \mathrm{nA}$, and the thermal noise coefficient was $1.8 \times 10^{-23} \mathrm{~W} / \mathrm{Hz}$ for each of the positive-intrinsic-negative (PIN) photodiodes at the detection part. Gaussian algorithm was used for BER estimation.

Figure 3 compares the variation of BER with respect to fiber length for the SPD against AND as well as modified-AND detection schemes. The SPD technique shows significant performance enhancement over other detection approaches at all fiber lengths and offers the advantage of increasing the length of the transmission link without affecting the QoS. Moreover, remarkably, the BER performance of the SPD technique at data rate of $1.25 \mathrm{Gbps}$ is identical to modified-AND detection technique at data rate of $622 \mathrm{Mbps}$. It is evident that the SPD tech- nique clearly enhances the throughput of SAC-OCDMA system.

\section{CONCLUSION}

In this contribution, a SPD technique has been proposed for incoherent SAC-OCDMA systems. The performance of the proposed method has been investigated both theoretically and by simulation. Compared to current detection approaches, the proposed technique can reduce BER greatly. It is shown that for 35 number of users transmitting signals simultaneously at data rate of $622 \mathrm{Mbps}$, the $\mathrm{BER}=1.4 \times 10^{-28}$ for SPD technique is much better compared to $9.3 \times 10^{-6}$ and $9.6 \times 10^{-3}$ for the modified-AND as well as the AND detection techniques, respectively. The enhanced performance afforded by the proposed technique is based on the elimination of PIIN and MAI in the optical domain. This allows requiring only a single photodiode to be used rather than two photodiodes as in typical subtraction detection approaches, leads to a low-cost receiver for each user. This implies the feasibility 
of SAC-OCDMA system based on SPD technique as a compelling candidate for implementation in future optical access networks.

\section{References}

[1] J. Penon, Z. A. El-Sahn, L. A. Rusch, and S. LaRochelle, "Spectralamplitude-coded OCDMA optimized for a realistic FBC frequency response," J. Lightwave Technol. 25, 1256-1263 (2007).

[2] J. A. Salehi, "Emerging OCDMA communication systems and data networks," J. Opt. Netw. 6, 1138-1178, (2007).

[3] E. D. J. Smith, R. J. Blaikie, and D. P. Taylor, "Performance enhancement of spectral-amplitude-coding optical CDMA using pulseposition modulation," IEEE Trans. Commun. 46, 1176-1185 (1998).

[4] A. D. McCoy, M. Ibsen, P. Horak, B. C. Thomsen, and D. J. Richardson, "Feasibility study of SOA-based noise suppression for spectral amplitude coded OCDMA," J. Lightwave Technol. 25, 394-401 (2007).

[5] S. Ayotte, M. Rochette, J. Magne, L. A. Rusch, and S. LaRochelle, "Experimental verification and capacity prediction of FE-OCDMA using superimposed FBG," J. Lightwave Technol. 23, 724-731 (2005).

[6] F. N. Hasoon, S. A. Aljunid, M. D. A. Samad, M. K. Abdullah, and S. Shaari, "Spectral amplitude coding OCDMA using AND subtraction technique," Appl. Optics 47, 1263-1268 (2008).
[7] D. Zaccarin, and M. Kavehrad, "An optical CDMA system based on spectral encoding of LED," IEEE Photon. Technol. Lett. 5, 479-482, (1993).

[8] H. M. R. Al-Khafaji, S. A. Aljunid, and H. A. Fadhil, "Improved BER based on intensity noise alleviation using developed detection technique for incoherent SAC-OCDMA systems," J. Mod. Optic. 59, 878-886, (2012).

[9] M. Rochette, S. Ayotte, and L. A. Rusch, "Analysis of the spectral efficiency of frequency-encoded OCDMA systems with incoherent sources," J. Lightwave Technol. 23, 1610-1619 (2005).

[10] S. A. Aljunid, M. Ismail, A. R. Ramli, B. M. Ali, and M. K. Abdullah, "A new family of optical code sequences for spectral-amplitudecoding optical CDMA systems," IEEE Photon. Technol. Lett. 16, 2383-2385 (2004).

[11] M. Noshad, and K. Jamshidi, "Bounds for the BER of codes with fixed cross correlation in SAC-OCDMA systems," J. Lightwave Technol. 29, 1944-1950 (2011).

[12] S.-P. Tseng, and J. Wu, "A new code family suitable for high-rate SAC OCDMA PONs applications," IEEE J. Select. Areas Commun. 28, 827-837 (2010). 